\title{
Influence of optical standing waves on the femtosecond laser-induced forward transfer of transparent thin films
}

\author{
David P. Banks, ${ }^{1, *}$, Kamal S. Kaur, ${ }^{1}$, and Robert W. Eason ${ }^{1}$ \\ ${ }^{1}$ Optoelectronics Research Centre, University of Southampton, Southampton SO17 1BJ, UK \\ *Corresponding author:dpb@orc.soton.ac.uk
}

\begin{abstract}
The effects of the formation of an optical standing wave during femtosecond laser-induced forward transfer of transparent films is analysed using a numerical interference model. The dependence of the intensity distribution on a number of easily-controllable experimental parameters is investigated. Results of the model are compared to experimental studies of the transfer of gadolinium gallium oxide $(\mathrm{GdGaO})$ with a polymer sacrificial layer. The model allows us to explain the observed variation in deposit morphology with the size of the air gap, and why forward transfer of the $\mathrm{GdGaO}$ was possible below the ablation thresholds of both polymer and oxide. (c) 2008 Optical Society of America
\end{abstract}

OCIS codes: $350.3390,190.4180,310.6860,160.2750,160.5470$

\section{Introduction}

The Laser-Induced Forward Transfer (LIFT) technique (as shown in fig. 1) was initially proposed by Bohandy et al. as a method for depositing metal for the repair of damaged photomasks [1]. In LIFT, a thin film of the material to be deposited (the donor film) is coated onto one face of a transparent support substrate (the carrier) and brought into close contact (typically the separation is on the order of a few microns) with another substrate (the receiver). Transfer of the donor film to the receiver is effected by focusing or demagnifying a laser pulse through the carrier onto the carrier-donor interface. The laser initiates melting [2] or ablation $[3,4]$ of the donor film, providing the thrust required to propel material to the receiver.

LIFT has been successfully applied for the direct-writing of metals (e.g., see [1,2,5-11] amongst others), polymers [12], oxides [13,14], superconductors [15], diamond [16], carbon nanotube field emission cathodes [17], conducting polymers [18], and an adenosine triphosphate sensor fabricated from luciferase [19]. Recently, several groups have also investigated 
the effects of using ultrashort (sub-picosecond) pulses for LIFT ( $f$ s-LIFT) [13, 20-24]. The majority of work has focused on achieving the smallest possible structures, but fs-LIFT has also been shown to be capable of transferring viable biomaterials [25] and contiguous solid-phase sections of donor film [26] without requiring a sacrificial matrix or protective layer.

\section{A. LIFT of transparent materials}

For materials that do not absorb strongly at the laser wavelength, other techniques have been developed that utilise ablation of absorbing sacrificial materials to provide the thrust for forward transfer [27-31]. Although these techniques have vastly expanded the range of materials that can be deposited using forward transfer, they also introduce extra complexity to the process. For example, one commonly applied approach is to include a sacrificial layer between the carrier and the donor, which is often referred to as a dynamic release layer (DRL) [28]. Metallic layers are often used as DRLs [28,29] but, as metals do not dissociate cleanly, contamination of deposited donor material by residual DRL is possible [32]. Although this problem can be minimised using optimised polymeric DRLs [30,33,34], such materials can limit the range of available donors due to their sensitivity to high temperatures or solvent treatment. Clearly, therefore, it would be desirable to have a technique capable of directly transferring transparent materials.

In a recent work, we demonstrated that by utilising multi-photon absorption of femtosecond duration pulses at $\lambda=800 \mathrm{~nm}$, forward transfer of an effectively transparent (absorption depth several orders of magnitude greater than the film thicknesses), amorphous gadolinium gallium oxide $(\mathrm{GdGaO})$ donor was possible [35]. Furthermore, by including a triazene polymer (TP) DRL [36], also transparent to the laser wavelength, it was possible to forward transfer very clean discs of $\mathrm{GdGaO}$ with no evidence of residual DRL. An interesting observation was that the transfer threshold using the DRL was only $\approx 20 \%$ of the TP ablation threshold at $\lambda=800 \mathrm{~nm}$.

\section{B. Optical standing waves}

An important consideration when transferring transparent films by fs-LIFT is the formation of an optical standing wave due to interference between forward and backward propagating waves reflecting off the various interfaces between carrier, DRL, donor, air gap and receiver. The result is potentially a large intensity modulation along the laser propagation direction within the thin films. Hence, the local intensity can vary significantly from the incident intensity, which will affect the transfer threshold and may cause unintentional damage to the transferred material.

In this work we will consider the fs-LIFT of transparent films in more detail. A model for 
the intensity profile in arbitrary stacks of DRL and donor film(s) is presented. The effects of various material and experimental parameters on the peak intensity within the film(s) are discussed in terms of their potential impact on the LIFT process. Lastly, the model is applied to the case of fs-LIFT of GdGaO with a TP-DRL. We investigate whether the formation of a standing wave can explain the surprisingly low transfer threshold observed previously [35]. Also studied are the effects of varying the size of the air-gap in the setup on the quality of the resultant $\mathrm{GdGaO}$ depositions.

\section{Standing wave model}

The formation of standing waves in thin films has been considered by a number of authors for the case of lithographic patterning (see e.g. [37]). In particular, an analytical description of the standing wave intensity pattern in stacks of arbitrary numbers of thin films has been developed by Mack [38].

\section{A. Single film case}

It is relatively simple to determine the intensity profile in a single thin film between two, effectively infinite, dielectric media, as shown in fig. 2. The film is exposed through layer 1 (refractive index, $\mathbf{n}_{\mathbf{1}}=n_{1}+i \kappa_{1}$ ) to a normally incident monochromatic plane wave, $\mathbf{E}_{\mathbf{I n}}(\mathbf{z})=$ $E_{I n} \exp \left(-i k_{1} z\right)$. For simplicity, it is chosen to set $z=0$ at the first interface, $\Gamma_{12}$. The wave is partially transmitted into the film where it undergoes consecutive reflections off $\Gamma_{23}$ and $\Gamma_{12}$. Note that, although the theory is for monochromatic illumination, the femtosecond Ti:sapphire laser used in the experiments (described later) had a bandwidth of around 10 $\mathrm{nm}$. The result of using a source with a finite bandwidth would be to reduce the standing wave amplitude relative to the monochromatic case. However, because the laser bandwidth was small compared to the standing wave period $(\approx 100) \mathrm{s} \mathrm{nm}$, the model still provided an acceptable level of accuracy for comparison between experimental and numerical results.

A geometrical approach yields a series of forward and backward propagating waves in the film, $\mathbf{E}^{+}(\mathbf{z})$ and $\mathbf{E}^{-}(\mathbf{z})$ respectively, of the form

$$
\begin{aligned}
& \mathbf{E}_{\mathbf{a}}^{+}(\mathbf{z})=E_{I n} t_{12} r_{23}^{a-1} r_{21}^{a-1} \tau_{2}^{2(a-1)} \exp \left(-i k_{2} z\right) \\
& \mathbf{E}_{\mathbf{a}}^{-}(\mathbf{z})=E_{I n} t_{12} r_{23}^{a} r_{21}^{a-1} \tau_{2}^{2 a} \exp \left(i k_{2} z\right)
\end{aligned}
$$

where $t_{i j}=2 \mathbf{n}_{i} /\left(\mathbf{n}_{i}+\mathbf{n}_{j}\right)=$ the amplitude transmission coefficient at $\Gamma_{i j}$

$r_{i j}=\left(\mathbf{n}_{i}-\mathbf{n}_{j}\right) /\left(\mathbf{n}_{i}+\mathbf{n}_{j}\right)=$ the amplitude reflection coefficient at $\Gamma_{i j}$

$\tau_{j}=\exp \left(-i k_{j} d_{j}\right)=$ the internal transmittance of layer $j$

$d_{j}=$ thickness of layer $j$

$k_{j}=2 \pi \mathbf{n}_{j} / \lambda=$ the propagation constant in layer $j$ 


$$
\begin{aligned}
& \lambda=\text { wavelength of incident laser pulse } \\
& a=\text { denotes multiple reflections }
\end{aligned}
$$

The total electric field in the thin film, $\mathbf{E}_{\mathrm{film}}(\mathbf{z})$, is then just the summation of all these forward and backward propagating waves:

$$
\mathbf{E}_{\mathrm{film}}(\mathbf{z})=\sum_{a=1}^{\infty}\left(\mathbf{E}_{\mathbf{a}}^{+}(\mathbf{z})+\mathbf{E}_{\mathbf{a}}^{-}(\mathbf{z})\right)
$$

Substituting eqns. (1) and (2) into (3), it is then straightforward to show that

$$
\mathbf{E}_{\mathbf{f i l m}}(\mathbf{z})=E_{I n} t_{12}\left[\exp \left(-i k_{2} z_{2}\right)+r_{23} \tau_{2}^{2} \exp \left(i k_{2} z_{2}\right)\right] \times S
$$

where $S=1+r_{21} r_{23} \tau_{2}^{2}\left(1+r_{21} r_{23} \tau_{2}^{2}(1+\ldots)\right)$, is a geometric series. Hence, noting that $r_{21}=-r_{12}$, we arrive at [38]

$$
\mathbf{E}_{\mathrm{film}}(\mathbf{z})=E_{\text {In }} t_{12}\left(\frac{\exp \left(-i k_{2} z\right)+r_{23} \tau_{2}^{2} \exp \left(i k_{2} z\right)}{1+r_{12} r_{23} \tau_{2}^{2}}\right)
$$

Finally, the intensity in the film, $\mathbf{I}_{\text {film }}(\mathbf{z})$, is found by squaring the magnitude of $\mathbf{E}_{\mathbf{f i l m}}(\mathbf{z})$,

$$
\mathbf{I}_{\text {film }}(\mathbf{z})=\frac{c \epsilon_{0} \epsilon_{2}}{2}\left|\mathbf{E}_{\text {film }}(\mathbf{z})\right|^{2}
$$

where $\epsilon_{0}$ is the permittivity of free space and $\epsilon_{2}$ is the permittivity of the thin film.

\section{B. Multiple film case}

The above analysis can be extended to the case of multiple thin films between two substrates. In this situation it is convenient to define two effective amplitude reflection coefficients, $r_{j, j+1}^{\prime}$ and $r_{j-1, j}^{*}$ [38]. Now $r_{j, j+1}^{\prime}$ is the effective reflection coefficient between layer $j$ and all the layers beyond it (i.e. layers $j+1, j+2, \ldots, m+1$ ) and $r_{j-1, j}^{*}$ is the coefficient between layer $j$ and all the preceding layers (i.e. layers $1,2, \ldots, j-1$ ). We also introduce the effective fluence incident on layer $j, E_{I n, j} \cdot r_{j-1, j}^{*}, r_{j, j+1}^{\prime}$, and $E_{I n, j}$ can be calculated from the refractive indices and thicknesses of all the layers in the structure as described in [38].

Consider now the case of $m-1$ thin films (i.e. $m+1$ layers including the two substrates). The substrates are layers 1 and $m+1$. The field in layer 2 , the first thin film, is then given by [38]:

$$
\mathbf{E}_{\mathbf{2}}(\mathbf{z})=E_{I n} t_{12} \frac{\exp \left(-i k_{2} z\right)+r_{23}^{\prime} \tau_{2}^{2} \exp \left(i k_{2} z\right)}{1+r_{12} r_{23}^{\prime} \tau_{2}^{2}}
$$

Note that the electric field in layer 2 is almost identical in the single and multiple film cases except that the reflection coefficient at $\Gamma_{23}$ in eqn. (5), $r_{23}$, is replaced by the effective reflection coefficient between layer 2 and all the subsequent layers, $r_{23}^{\prime}$. 
Similarly, if it is desired to know the field in the $j^{\text {th }}$ layer, $\mathbf{E}_{\mathbf{j}}(z)$, this is given by [38]

$$
\mathbf{E}_{\mathbf{j}}(\mathbf{z})=E_{I n, j} t_{j-1, j}^{\prime} \frac{\exp \left(-i k_{j} z_{j}\right)+r_{j, j+1}^{\prime} \tau_{j}^{2} \exp \left(i k_{j} z_{j}\right)}{1+r_{j-1, j}^{*} r_{j, j+1}^{\prime} \tau_{j}^{2}}
$$

where $z_{j}$ is the local depth in layer $j$, such that $z_{j}=0$ at $\Gamma_{j-1, j}$ and $z_{j}=d_{j}$ at $\Gamma_{j, j+1}$, and $t_{j-1, j}^{\prime}$ is the effective transmission coefficient at $\Gamma_{j-1, j}\left(t_{j-1, j}^{\prime}=1-r_{j-1, j}^{\prime}=1+r_{j-1, j}^{*}\right)$. Note that, in layer $m$ (the last thin film), $r_{j, j+1}^{\prime}$ is just equal to $r_{j, j+1}$.

The intensity in the top or $j^{\text {th }}$ layer can then be found by squaring the magnitude of eqn. (7) or (8), respectively.

$$
\begin{aligned}
& \mathbf{I}_{\mathbf{2}}(\mathbf{z})=\frac{c \epsilon_{0} \epsilon_{2}}{2}\left|\mathbf{E}_{\mathbf{2}}(\mathbf{z})\right|^{2} \\
& \mathbf{I}_{\mathbf{j}}(\mathbf{z})=\frac{c \epsilon_{0} \epsilon_{j}}{2}\left|\mathbf{E}_{\mathbf{j}}(\mathbf{z})\right|^{2}
\end{aligned}
$$

where $\epsilon_{j}$ is the permittivity of layer $j$.

\section{Implications of Standing Waves for fs-LIFT}

Using the above, multi-layered model, it is simple to determine the standing wave intensity profile in a typical fs-LIFT setup. Consider first the case of a single donor film of refractive index $\mathbf{n}_{\text {donor }}$ and thickness $d_{\text {donor }}$ on a silica carrier $\left(\mathbf{n}_{\text {carrier }} \approx 1.47+0 i\right)$ separated by an air gap of thickness $d_{\text {air }}$ from a receiver with a surface reflection coefficient $r_{\text {rec }}$, as shown in fig. 3. Clearly the carrier is equivalent to layer 1 in the above model; the donor is layer 2 , the air gap layer 3 , and the receiver layer 4 .

For the simulations, the laser wavelength was chosen to be $800 \mathrm{~nm}$, in accordance with experiments. $E_{I n}, I_{2}$, and $I_{j}$ were all set to 1 for simplicity, and hence the incident intensity at the carrier-donor interface $(z=0)$ was also unity. For the donor, a material similar to the $\mathrm{GdGaO}$ was chosen, so $\mathbf{n}_{\text {donor }}=n_{\text {donor }}+i \kappa_{\text {donor }} \approx 1.95-0.03 i$; the value of $\kappa_{\text {donor }}$ corresponded to a $1 / e$ absorption depth of approximately $2 \mu \mathrm{m}$.

There are essentially three variable parameters in this set-up, $d_{\text {donor }}, d_{\text {air }}$, and $r_{\text {rec }}\left(\mathbf{n}_{\text {donor }}\right.$ is obviously fixed by the application). Figure 4 shows the variation of maximum intensity in the donor, $I_{(\text {donor,Max })}$, as a function of $d_{\text {air }}$ for a range of donor film thicknesses, with receivers approximately corresponding to silica $\left(r_{r e c}=-0.2\right.$, fig. $\left.4(\mathrm{a})\right)$ and silicon $\left(r_{r e c}=-0.57\right.$, fig. $4(\mathrm{~b}))$. The most obvious trend that can be seen is that $I_{(\text {donor,Max })}$ varied approximately sinusoidally with $d_{\text {air }}$ with a period of around $\lambda / 2$. This result was intuitive as interference in the donor film due to the first reflection off the receiver was constructive for $2 d_{\text {air }}=$ $\lambda / 2,3 \lambda / 2, \ldots$ and destructive for $2 d_{\text {air }}=0, \lambda, 2 \lambda, \ldots$ (note the $\pi$-phase shift upon reflection from the air-receiver interface).

The dependence on $d_{\text {donor }}$ was also relatively easy to explain. Due to interference between the forward propagating incident wave and the two back-reflections off the donor-air interface 
and the receiver surface, a pattern of maxima and minima was formed in the donor film with a period of $\lambda / 2 n_{\text {donor }} \approx 200 \mathrm{~nm}$. Consider fig. 5 which shows the calculated intensity profiles in the donor and air layers with $\left(d_{\text {donor }}(\mu m), d_{\text {air }}(\mu m)\right)$ equal to $(0.1,0.125),(0.1,0.375)$, $(0.5,0.2)$, and $(0.5,0.4)$, in fig. $5(\mathrm{a})-(\mathrm{d})$, respectively. The receiver reflectivity was $r_{r e c}=-0.57$ and the refractive index profile is included as a dashed line to indicate the donor-air boundary.

The necessity to have an intensity minimum at the receiver surface meant that $d_{\text {air }}$ determined the positions of the maxima and minima in the donor layer. For very thin films $\left(d_{\text {donor }} \leq \lambda / 2 n_{\text {donor }}\right)$, the film thickness was less than the standing wave period so whether there was a maximum or minimum in the donor was determined by $d_{\text {air }}$ (see fig. $5(\mathrm{a})$ and (b)). As a result, the maximum intensity in very thin films was highly dependent on $d_{\text {air }}$ (see fig. 4). For thicker films with $d_{\text {donor }}>\lambda / 2 n_{\text {donor }}$, there was always a standing wave maximum in the donor, and so the dependence on $d_{\text {air }}$ decreased. However, there was still a significant, approximately sinusoidal, dependence of the maximum on $d_{\text {air }}$.

LIFT techniques are well known to be highly sensitive to the applied laser fluence (see e.g. $[1,21])$. Hence, having the maximum intensity in the donor highly dependent on $d_{\text {air }}$ represents a problem as $d_{\text {air }}$ is then another parameter that must be accurately controlled for reproducible results. If we define $\beta$ as the ratio of the maximum value of $I_{\text {donor,Max }}$ achieved by varying $d_{\text {air }}$ to the minimum value, then $\beta$ quantifies the variation of the maximum intensity in the donor as $d_{\text {air }}$ is changed. A value of $\beta=1$ is ideal so there is no dependence of $I_{\text {donor,Max }}$ on $d_{\text {air }}$ and critical control of the size of the air gap is not necessary.

Figure 6 shows plots of $\beta$ as functions of $r_{\text {rec }}$ (a) and $d_{\text {donor }}$ (b) with all other parameters fixed. There were clearly strong dependences on both parameters. For receivers with very low reflection coefficients (e.g. silica, $r_{r e c}=-0.2$ ), the back-reflection off the receiver was comparatively small compared to the standing wave in the film due to the strong backreflection off the donor-air interface. Hence, the variation of $\beta$ with $d_{\text {donor }}$ was also small (fig. 6 (a)).

As $\left|r_{r e c}\right|$ was increased, the influence of the back reflection off the receiver was enhanced, and hence the value of $\beta$ also began to rise. For very thin films (with $d_{\text {donor }} \leq \lambda / 2 n_{\text {donor }}$ as before), the increase in $\beta$ was monotonic due to the lack of the formation of complete fringes in the donor (fig. $6(\mathrm{~b}))$. For thicker donors $\left(d_{\text {donor }} \geq \lambda / 2 n_{\text {donor }}\right), \beta$ increased to a peak at $r_{r e c} \approx-0.35$ (fig. $6(\mathrm{~b})$ ). The value of $r_{\text {rec }}$ at which this peak occurred was observed to be virtually independent of $d_{d o n o r}$, but strongly dependent on $\mathbf{n}_{\text {donor }}$. The origin of the peak was due to the relative amplitudes of the back-reflections from the donor-air interface and the receiver. A wave passing through the donor-air interface, reflecting off the receiver and then re-entering the donor layer experienced an effective reflection coefficient, $r_{e f f}$, of $t_{23} r_{\text {rec }} t_{32}$. A simple calculation then yields $r_{r e c} \approx-0.35$ corresponds to $r_{\text {eff }} \approx r_{23}$. Hence, interference was almost completely destructive for $2 d_{\text {air }}=0, \lambda, 2 \lambda, \ldots$, and fully constructive 
for $2 d_{\text {air }}=\lambda / 2,3 \lambda / 2, \ldots$ when $r_{\text {rec }} \approx-0.35$. This led to the largest possible variation of $I_{\text {donor, Max }}$ with $d_{\text {air }}$, and hence a maximum in the value of $\beta$.

For $r_{r e c} \geq r_{\text {eff }}$, the value of $\beta$ began to fall to a minimum (fig. 6 (b)). Note that, for certain values of $d_{\text {donor }}$, the minimum was at an unphysical value of $\left|r_{r e c}\right|>1$. To understand this behaviour, it is necessary to consider the higher-order reflections that can occur. For example, consider the second order reflection off the donor-carrier interface for backward-propagating waves in the donor. The relative strength of this reflection will depend on whether there is a minimum or a maximum at this interface, which in turn depends on whether there is a minimum or a maximum at the donor-air interface. The important point to note is that, if $d_{\text {donor }}=m \lambda / 4 \mathbf{n}_{\text {donor }}(m=1,3,5, \ldots)$ then there will always be a minimum at the donor-carrier interface if there is a maximum at the donor-air interface and vice-versa (see fig. $5(\mathrm{c}) \&(\mathrm{~d}))$. For $d_{\text {donor }}=m \lambda / 4 \mathbf{n}_{\text {donor }}(m=2,4,6, \ldots)$ the opposite is true. Hence, for $m=1,3,5, \ldots$, the reflection off the donor-carrier interface acts to counter the variation in $I_{\text {donor,Max }}$ due to $d_{\text {air }}$, whilst for $m=2,4,6, \ldots$ the two effects combine to increase the variation. Thus the second-order reflection explains why a periodic behaviour of $\beta$ with $d_{\text {donor }}$ is observed with a period of $\lambda / 2 \mathbf{n}_{\text {donor }}$ (fig. 6 (a)). Similarly, the reason why $\beta$ begins to increase again above a certain $d_{d o n o r}$-dependent value of $\left|r_{r e c}\right|$ can be explained by considering even higher order reflections. One final observation to note is that, for all values of $r_{r e c}, \beta \rightarrow 1$ as $d_{\text {donor }} \rightarrow \infty$ due to the small amount of absorption in the donor film.

To conclude this section, fs-LIFT of transparent films is complicated by the formation of an optical standing wave. The effect of this standing wave is a dependence of $I_{d o n o r, M a x}$ on $d_{\text {air }}$ that is not observed with absorbing films and means that $d_{\text {air }}$ must be accurately controlled to achieve reproducible results. However, using the above model, it is possible to identify sets of experimental parameters that minimise this problem. If the application allows for variation of $r_{r e c}$, then the problem can be reduced significantly. If only control of $d_{\text {air }}$ and $d_{\text {donor }}$ is available, as is likely in many real applications, then it is still possible to use the model to find optimal values of these parameters.

\section{4. fs-LIFT of transparent GdGaO: Influence of $d_{\text {air }}$}

In the previous section it was demonstrated theoretically that the formation of an optical standing wave during fs-LIFT of transparent films led to a strong dependence of the peak intensity in the film(s) on the size of the air-gap. It was shown that great care must be taken to choose optimal values of the various experimental parameters to obtain reproducible results. In this section we will consider how significant the effect is in practice by investigating the transfer of a sample transparent donor and how the deposited structures on the receiver vary with $d_{\text {air }}$.

As a sample donor material, a GdGaO film transparent to all wavelengths greater than 
$\approx 300 \mathrm{~nm}$ was used. In a recent work we demonstrated the fs-LIFT of this material using multi-photon absorption of an $800 \mathrm{~nm}$ Ti:sapphire laser pulse (130 fs) [35]. However, it was observed that direct transfer of the donor resulted in poor quality depositions, which would be difficult to analyse in terms of damage caused by localised high intensities corresponding to standing wave maxima. By including a TP-DRL, transparent to wavelengths longer than $400 \mathrm{~nm}$, it was possible to transfer clean discs of GdGaO. Hence we will use the same setup already described in [35] (DRL: TP $100 \mathrm{~nm}$ thick; donor: GdGaO $150 \mathrm{~nm}$ thick) for our studies here.

The above model can easily be extended to include the DRL by including an extra layer between the carrier and the donor. The TP-DRL is now layer 2 with refractive index $\mathbf{n}_{\mathbf{D R L}}=$ $n_{D R L}+i \kappa_{D R L} \approx 1.7-0.06 i[39]$; the $\mathrm{GdGaO}$ donor is layer 3, the air layer 4, and the receiver layer 5. Note that, in the previous section the value of $n_{\text {donor }}$ was taken to be 1.95 . This corresponds to the refractive index of single crystal gadolinium gallium garnet. However, because the donor in this experiment had to be grown at room temperature to protect the heat-sensitive TP-DRL, the resultant film was amorphous. The refractive index of this amorphous $\mathrm{GdGaO}$ is not known so it was chosen to use the value of $n_{\text {donor }}=1.95$ as an approximation.

In this multiple-film geometry, the parameter $\beta$ is important in both the DRL and donor films. Similar calculations to those performed above again yielded periodic dependences of $\beta$ on the film thicknesses of both DRL and donor, as shown in fig. 7(a) and (b), respectively. This behaviour can be easily explained considering the relative intensities of the multiple reflections as before.

Assuming the best guesses of $n_{D R L}$ and $n_{\text {donor }}$ given above for the TP and the GdGaO, the model gives $\beta=3.0717$ in the DRL and $\beta=1.7882$ in the donor for $d_{D R L}=100 \mathrm{~nm}$ and $d_{\text {donor }}=150 \mathrm{~nm}$. When $n_{D R L}$ and $n_{\text {donor }}$ were allowed to vary while keeping the film thicknesses constant (fig. 7(c)), it was found that, although there were dependences of $\beta$ on the refractive indices in both layers, these dependences were comparatively weak. Hence, the most important parameters in determining the values of $\beta$ in the two films appear to be the film thicknesses. In this case the minimum values of $\beta$ were $\approx 1.25$ and $\approx 1.4$ in the DRL and donor layers, respectively. However, given the uncertainty in the values of $\mathbf{n}_{\mathbf{D R L}}$ and $\mathbf{n}_{\text {donor }}$, such numerical results should be considered as somewhat approximate.

\section{A. Experiments with $G d G a O$}

The fs-LIFT experiments were performed using a micromachining workstation (modified New Wave UP-266) fitted with a white light source and a CCD camera for viewing the workpiece. As the DRL and donor films were transparent to the white light, thin film interference of the white light occurred that was observed as coloured fringes on the CCD. Estimating the 
reflection coefficients of the interfaces using the previously given refractive indices yielded $r_{12} \approx-0.07, r_{23} \approx-0.07, r_{34} \approx 0.32$, and $r_{45}=r_{r e c} \approx-0.57$. Hence, the back reflections off the donor-air and air-receiver interfaces were by far the strongest and so we can conclude that the visible fringes were primarily the result of thin film interference in the air gap. Therefore, it was possible to estimate $d_{a i r}$ from the colour of the fringe, $\lambda_{f}$, and the fringe number, $f$. For example, for the first green fringe, $\lambda_{f} \approx 530$ and $f=1, d_{\text {air }}=f \lambda_{f} / 4 \approx 130$ nm.

Figure 8 shows SEM micrographs of deposited GdGaO as a function of $d_{\text {air }}$. For reasons described in [35], it is believed that all the TP-DRL was removed during the forward transfer process. Observation of these results revealed a periodic dependence of the GdGaO deposit morphology on $d_{\text {air }}$. With donor and receiver in tight contact $\left(d_{\text {air }} \approx 0\right.$ fig. 8(a)) no deposits were obtained. This was believed to be due to the fact that the donor layer did not have sufficient room to deform and shear due to the close proximity of the receiver [35]. Increasing $d_{\text {air }}$ to around 50 or $100 \mathrm{~nm}$ (fig. 8(b)) resulted in the good quality, unshattered discs reported previously [35]. For $d_{\text {air }} \approx 200 \mathrm{~nm}$, the deposited structures were very different, exhibiting significant laser damage and splattered material transferred around the main deposit (fig. 8(c)). Further increasing $d_{\text {air }}$ resulted in a gradual reduction in the amount of apparent laser damage (fig. 8(d)) until around $d_{\text {air }} \approx 400 \mathrm{~nm}$ when similar good quality discs to those observed at $d_{\text {air }} \leq 100 \mathrm{~nm}$ were seen again (fig. 8(e)). However, at this separation, there was evidence of more (solid phase) debris, believed to be due to shattering during transfer [35].

When $d_{\text {air }}$ was increased to greater than $400 \mathrm{~nm}$, similar behaviour was observed. Firstly, the amount of laser damage to the GdGaO increased with $d_{\text {air }}$ up to $d_{\text {air }} \approx 600 \mathrm{~nm}$ (fig. 8(f)), before decreasing again until relatively clean discs were seen for $d_{\text {air }} \approx 800 \mathrm{~nm}$ (fig. $8(\mathrm{~g})$ ). This periodic variation of deposits continued with the donor-receiver separation, with goodquality deposits obtained when $d_{\text {air }} \approx 400,800, \ldots \mathrm{nm}$ and significantly damaged material when $d_{\text {air }} \approx 200,600, \ldots \mathrm{nm}$.

It is simple to explain the observed variation in the quality of deposited material by considering the effect of multiple reflections. In previous work, the forward transfer threshold of a $150 \mathrm{~nm} \mathrm{GdGaO}$ donor on a $100 \mathrm{~nm}$ TP-DRL was measured to be around $90 \mathrm{~mJ} / \mathrm{cm}^{2}$, compared to ablation thresholds of 110 and $500 \mathrm{~mJ} / \mathrm{cm}^{2}$ for the $\mathrm{GdGaO}$ and TP, respectively [35]. Figure 9 shows a plot of the variation of maximum fluence in the DRL (solid line) and donor (dashed line) layers with $d_{a i r}$ calculated using the material properties described above and assuming an incident fluence of $90 \mathrm{~mJ} / \mathrm{cm}^{2}$. The SEM micrographs A-G show typical deposits obtained at values of $d_{\text {air }}$ indicated by $\mathrm{A}-\mathrm{G}$ on the graph. As would be expected, the relatively undamaged deposits coincided with values of $d_{\text {air }}$ where destructive interference resulted in relatively low fluences in the DRL and donor (see A,D,G). Similarly, the most obviously damaged deposits were obtained when $d_{\text {air }}$ corresponded to constructive 
interference in the films $(\mathrm{B}, \mathrm{E})$.

The results in fig. 9 also provide a likely explanation for the surprisingly low (below the ablation threshold) transfer thresholds of GdGaO when a TP-DRL was included [35]. As can be seen from the plot in fig. 9, assuming an incident fluence of $90 \mathrm{~mJ} / \mathrm{cm}^{2}$, the maximum fluence in the TP-DRL varied from $\approx 80$ to $\approx 250 \mathrm{~mJ} / \mathrm{cm}^{2}$ as $d_{\text {air }}$ changed, and hence the fluence was never sufficient to photolytically decompose the TP-DRL. However, the fluence in the donor was consistently above $110 \mathrm{~mJ} / \mathrm{cm}^{2}$ (i.e. greater than the measured ablation threshold). This observation suggested that the forward transfer of GdGaO resulted from absorption of the laser in, and associated heating of, the donor; the hot donor film then conductively heated the TP-DRL which subsequently thermally decomposed. Note that these results indicate that it is possible to get good quality transfer of donor material, even when the donor has a lower ablation threshold than the DRL at the laser wavelength, provided that the donor can withstand heating to the DRL ablation/decomposition temperature. Note also the apparent disparity between the numerical results in fig. 9 and the measured ablation threshold of the GdGaO. The model predicted fluences in the donor well above its ablation threshold for most values of $d_{a i r}$, but no evidence of laser damage was seen in the deposits at $d_{\text {air }} \approx 0,400,800, \ldots \mathrm{nm}$. This can be explained by the fact that the model does not take into account the increase in $\kappa_{\text {donor }}$ as the fluence approached the ablation threshold and the GdGaO began to absorb the laser nonlinearly.

\section{Conclusions}

To conclude, a thin film interference model has been used to predict the standing wave intensity distribution formed during the femtosecond LIFT of transparent films. The model has been used to investigate the effects of varying a number of experimental parameters, including the thicknesses of the donor, DRL, and air layers, and the receiver reflectivity. An important consideration that has been identified was the dramatic variation of the fluence in the DRL and donor layers as $d_{\text {air }}$ was changed. We have shown that, in principle, it is possible to minimise this effect with the proper choice of film thicknesses (and receiver reflectivity, if this parameter can be varied).

The results of the model have been compared to experimental results of the transfer of a GdGaO donor on a TP-DRL. The model appeared to explain the periodic variation in the morphology of $\mathrm{GdGaO}$ deposits with $d_{\text {air }}$. Also, the model provided an explanation of the below ablation threshold transfer of GdGaO. The transfer process is now believed to be a thermolytic decomposition of the TP layer due to conductive heating from the overlying donor, which nonlinearly absorbed the laser at a lower fluence. Taken together, the experimental and numerical results indicate that fs-LIFT is a viable method for the transfer of transparent materials. However, extra care must be taken with regards to the formation 
of a standing wave and the relative damage thresholds of the DRL and donor materials.

\section{Acknowledgments}

This work was funded by the Engineering and Physical Sciences Research Council, UK, under Grant No. EP/C515668/1. The authors are grateful to Romain Fardel and Matthias Nagel at EMPA, and Thomas Lippert at the Paul Scherrer Institut for providing the TP-DRLs and for illuminating discussions. 


\section{References}

1. J. Bohandy, B. Kim, and F. Adrian, "Metal deposition from a supported metal film using an excimer laser," J. Appl. Phys. 60, 1538-1539 (1986).

2. D. Willis and V. Grosu, "Microdroplet deposition by laser-induced forward transfer," Appl. Phys. Lett. 86, 244103 (2005).

3. F. Adrian, J. Bohandy, B. Kim, A. Jette, and P. Thompson, "A study of the mechanism of metal deposition by the laser-induced forward transfer process," J. Vac. Sci. Technol. B 5, 1490-1494 (1987).

4. R. Baseman and N. Froberg, "Minimum fluence for laser blow-off of thin gold films at 248 and 532 nm," Appl. Phys. Lett. 56, 1412-1414 (1990).

5. Z. Toth, Z. Kantor, P. Mogyorosi, and T. Szorenyi, "Surface patterning by pulsed laser induced transfer of metals and compounds," Proc. SPIE 1279, 150-157 (1990).

6. H. Esrom, J. Zhang, U. Kogelschatz, and A. Pedraza, "New approach of a laser-induced forward transfer for deposition of patterned thin metal films," Appl. Surf. Sci. 86, 202207 (1995).

7. I. Zergioti, D. Papazoglou, A. Karaiskou, C. Fotakis, E. Gamaly, and A. Rode, "A comparative schlieren imaging study between ns and sub-ps laser forward transfer of Cr," Appl. Surf. Sci. 208-209, 177-180 (2003).

8. Y. Nakata and T. Okada, "Time-resolved microscopic imaging of the laser-induced forward transfer process," Appl. Phys. A [Suppl.] 69, S275-S278 (1999).

9. R. Bahnisch, W. Gross, and A. Menschig, "Single-shot, high repetition rate metallic pattern transfer," Microelectron. Eng. 50, 541-546 (2000).

10. B. Tan, K. Venkatakrishnan, and K. Tok, "Selective surface texturing using femtosecond pulsed laser induced forward transfer," Appl. Surf. Sci. 207, 365-371 (2003).

11. L. Landstrom, J. Klimstein, G. Schrems, K. Piglmayer, and D. Bauerle, "Single-step patterning and the fabrication of contact masks by laser-induced forward transfer," Appl. Phys. A 78, 537-548 (2004).

12. I. Lee, W. Tolbert, D. Dlott, M. Doxtader, D. Foley, D. Arnold, and E. Ellis, "Dynamics of laser ablation transfer imaging investigated by ultrafast microscopy," J. Imag. Sci. Tech. 36, 180-187 (1992).

13. I. Zergioti, S. Mailis, N. Vainos, P. Papakonstantinou, C. Kalpouzos, C. Grigoropoulos, and C. Fotakis, "Microdeposition of metal and oxide structures using ultrashort laser pulses," Appl. Phys. A 66, 579-582 (1998).

14. H. Sakata, S. Chakraborty, E. Yokoyama, M. Wakaki, and D. Chakravorty, "Laserinduced forward transfer of tio-au nanocomposite films for maskless patterning," Appl. Phys. Lett. 86, 114104 (2005). 
15. E. Fogarassy, C. Fuchs, F. Kerherve, G. Hauchecorne, and J. Perriere, "Laser-induced forward transfer of high- $T_{c}$ ybacuo and bisrcacuo superconducting thin films," J. Appl. Phys. 66, 457-459 (1989).

16. S. Pimenov, G. Shafeev, A. Smolin, V. Konov, and B. Vodolaga, "Laser-induced forward transfer of ultra-fine diamond particles for selective deposition of diamond films," Appl. Surf. Sci. 86, 208-212 (1995).

17. S. Chang-Jian, J. Ho, J. Cheng, and C. Sung, "Fabrication of carbon nanotube field emission cathodes in patterns by a laser transfer method," Nanotechnology 17, 11841187 (2006).

18. B. Thomas, A. Alloncle, P. Delaporte, M. Sentis, S. Sanaur, M. Barret, and P. Collot, "Experimental investigations of laser-induced forward transfer process of organic thin films," Appl. Surf. Sci. 254, 1206-1210 (2007).

19. Y. Tsuboi, Y. Furuhata, and N. Kitamura, "A sensor for adenosine triphosphate fabricated by laser-induced forward transfer of luciferase onto a poly(dimethylsiloxane) microchip," Appl. Surf. Sci. 253, 8422-8427 (2007).

20. L. Yang, C. Wang, X. Ni, Z. Wang, W. Jia, and L. Chai, "Microdroplet deposition of copper film by femtosecond laser-induced forward transfer," Appl. Phys. Letts 89, 161110 (2006).

21. D. Banks, C. Grivas, J. Mills, I. Zergioti, and R. Eason, "Nanodroplets deposited in microarrays by femtosecond ti:sapphire laser induced forward transfer," Appl. Phys. Lett. 89, 193107 (2006).

22. S. Bera, A. Sabbah, J. Yarbrough, C. Allen, B. Winters, C. Durfee, and J. Squier, "Optimization study of the femtosecond laser-induced forward-transfer process with thin aluminium films," Appl. Optics 46, 4650-4659 (2007).

23. C. Germain, L. Charron, L. Lilge, and Y. Tsui, "Electrodes for microfluidic devices produced by laser induced forward transfer," Appl. Surf. Sci. 253, 8328-8333 (2007).

24. F. Claeyssens, A. Klini, A. Mourka, and C. Fotakis, "Laser patterning of zn for zno nanostructure growth: Comparison between laser induced forward transfer in air and in vacuum," Thin Solid Films 515, 8529-8533 (2007).

25. I. Zergioti, A. Karaiskou, D. Papazoglou, C. Fotakis, M. Kapsetaki, and D. Kafetzopoulos, "Time resolved schlieren study of sub-pecosecond and nanosecond laser transfer of biomaterials," Appl. Surf. Sci. 247, 584589 (2005).

26. D. Banks, C. Grivas, I. Zergioti, and R. Eason, "Ballistic laser-assisted solid transfer (blast) from a thin film precursor," Opt. Express 16, 3249-3254 (2008).

27. A. Pique, D. Chrisey, R. Auyeung, J. Fitz-Gerald, H. Wu, R. McGill, S. Lakeou, P. Wu, V. Nguyen, and M. Duignan, "A novel laser transfer process for direct writing of electronic and sensor materials," Appl. Phys. A [Suppl.] 69, S279-S284 (1999). 
28. W. Tolbert, I. Lee, M. Doxtader, E. Ellis, and D. Dlott, "High-speed color imaging by laser ablation transfer with a dynamic release layer: fundamental mechanisms," J. Imag. Sci. Tech. 37, 411-421 (1993).

29. P. Serra, M. Colina, J. Fernandez-Pradas, L. Sevilla, and J. Morenza, "Preparation of functional dna microarrays through laser-induced forward transfer," Appl. Phys. A 85, 1639-1641 (2004).

30. G. Blanchet, Y.-L. Loo, J. Rogers, F. Gao, and C. Fincher, "Large area, high resolution, dry printing of conducting polymers for organic electronics," Appl. Phys. Lett. 82, 463465 (2003).

31. R. Fardel, M. Nagel, F. Nuesch, T. Lippert, and A. Wokaun, "Fabrication of organic light-emitting diode pixels by laser-assisted forward transfer," Appl. Phys. Lett. 91, 061103 (2007).

32. T. Smausz, B. Hopp, G. Kecskemeti, and Z. Bor, "Study on metal microparticle content of the material transferred with absorbing film assisted laser induced forward transfer when using silver absorbing layer," Appl. Surf. Sci. 252, 4738-4742 (2006).

33. R. Fardel, M. Nagel, F. Nuesch, T. Lippert, and A. Wokaun, "Laser forward transfer using a sacrificial layer: influence of the material properties," Appl. Surf. Sci. 254, 13221326 (2007).

34. N. Kattamis, P. Purnick, R. Weiss, and C. Arnold, "Thick film laser induced forward transfer for deposition of thermally and mechanically sensitive materials," Appl. Phys Lett. 91, 171120 (2007).

35. D. Banks, K. Kaur, R. Gazia, R. Fardel, M. Nagel, T. Lippert, and R. Eason, "Triazene photopolymer dynamic release layer-assisted femtosecond laser-induced forward transfer with an active carrier substrate," Europhys. Lett. 83, 38003 (2008).

36. M. Nagel, R. Hany, T. Lippert, M. Molberg, F. Nuesch, and D. Rentsch, "Aryltriazene photopolymers for uv-laser applications: Improved synthesis and photodecomposition study," Macromol. Chem. Phys. 208, 277-286 (2007).

37. F. Dill, "Optical lithography," IEEE T. Electron Dev. ED-22, 440-444 (1975).

38. C. Mack, "Analytical expression for the standing wave intensity in photoresist," Appl. Optics 25, 1958-1961 (1986).

39. J. Bonse, J. Solis, L. Urech, T. Lippert, and A. Wokaun, "Femtosecond and nanosecond laser damage thresholds of doped and undoped triazenepolymer thin films," Appl. Surf. Sci. 253, 7787-7791 (2007). 


\section{List of Figure Captions}

Fig. 1. Schematic of the LIFT technique.

Fig. 2. Schematic of multiple reflections in a single transparent thin film under monochromatic, plane-wave illumination. Multiple reflections have been spatially separated for ease of viewing. $\Gamma_{12}$ and $\Gamma_{23}$ represent the interfaces between layers 1 and 2 , and 2 and 3 , respectively. Fig. 3. Fs-LIFT of a single film.

Fig. 4. Variation of the maximum intensity in the donor with $d_{a i r}$ and $d_{\text {donor }}$ assuming $r_{\text {rec }}=-0.2(\mathrm{a})$ and $-0.57(\mathrm{~b})$.

Fig. 5. Intensity (solid lines) and refractive index (dashed lines) profiles with $\left(d_{\text {donor }}(\mu m), d_{\text {air }}(\mu m)\right)$ equal to $(0.1,0.125),(0.1,0.375),(0.5,0.2)$, and $(0.5,0.4)$, in $(\mathrm{a}-\mathrm{d})$, respectively.

Fig. 6. Plots of $\beta$ as functions of $d_{\text {donor }}$ (a) and $r_{\text {rec }}$ (b) with other parameters fixed.

Fig. 7. Plots of $\beta$ in the TP-DRL (a) and in the GdGaO donor (b) as functions of $d_{\text {donor }}$ and $d_{D R L}$ with other parameters fixed, and variation of $\beta$ in the DRL (diamonds) and donor (circles) layers as function of $n_{D R L}$ and $n_{\text {donor }}$ (c).

Fig. 8. SEM micrographs of $\mathrm{GdGaO}$ deposits on $\mathrm{Si}$ as a function of $d_{\text {air }}$ transferred with incident laser fluence $\approx 90 \mathrm{~mJ} / \mathrm{cm}^{2}$.

Fig. 9. Main graph: variation of maximum fluence in TP-DRL (solid line) and GdGaO donor (dashed line) as functions of $d_{\text {air }}$ assuming incident fluence $90 \mathrm{~mJ} / \mathrm{cm}^{2}$. SEM micrographs show deposited material at approximately indicated separations; scale bars represent $10 \mu \mathrm{m}$. 


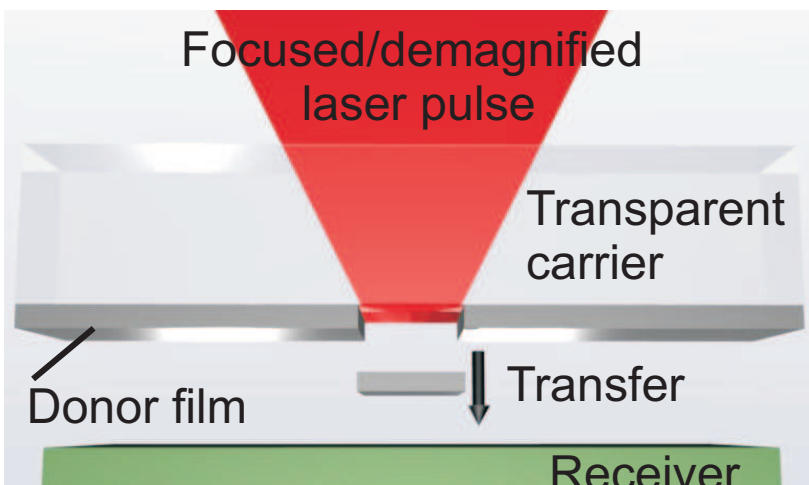

Fig. 1. Schematic of the LIFT technique. 


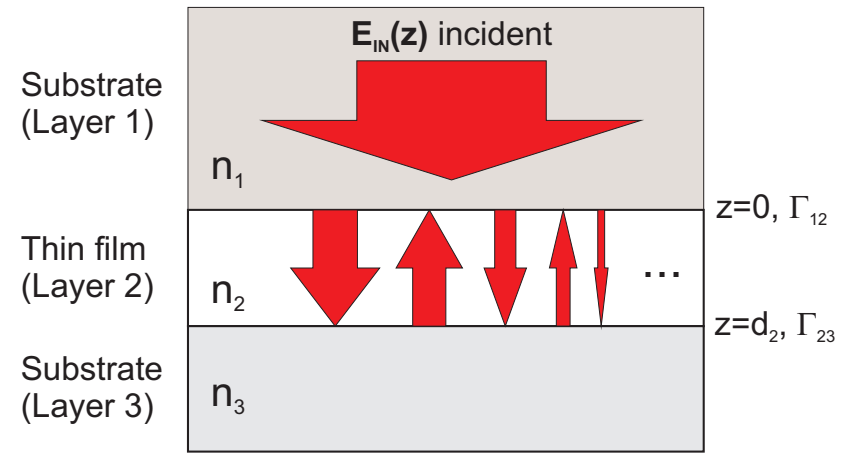

Fig. 2. Schematic of multiple reflections in a single transparent thin film under monochromatic, plane-wave illumination. Multiple reflections have been spatially separated for ease of viewing. $\Gamma_{12}$ and $\Gamma_{23}$ represent the interfaces between layers 1 and 2 , and 2 and 3, respectively. 


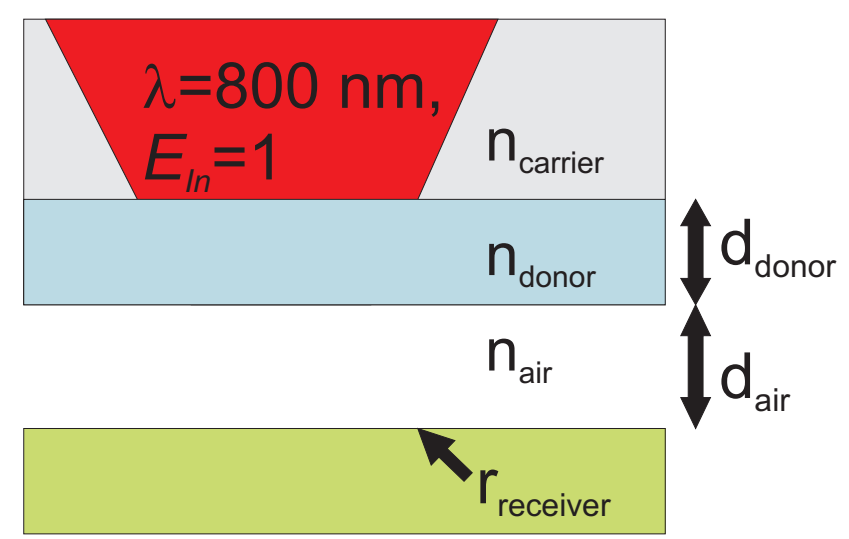

Fig. 3. Fs-LIFT of a single film. 

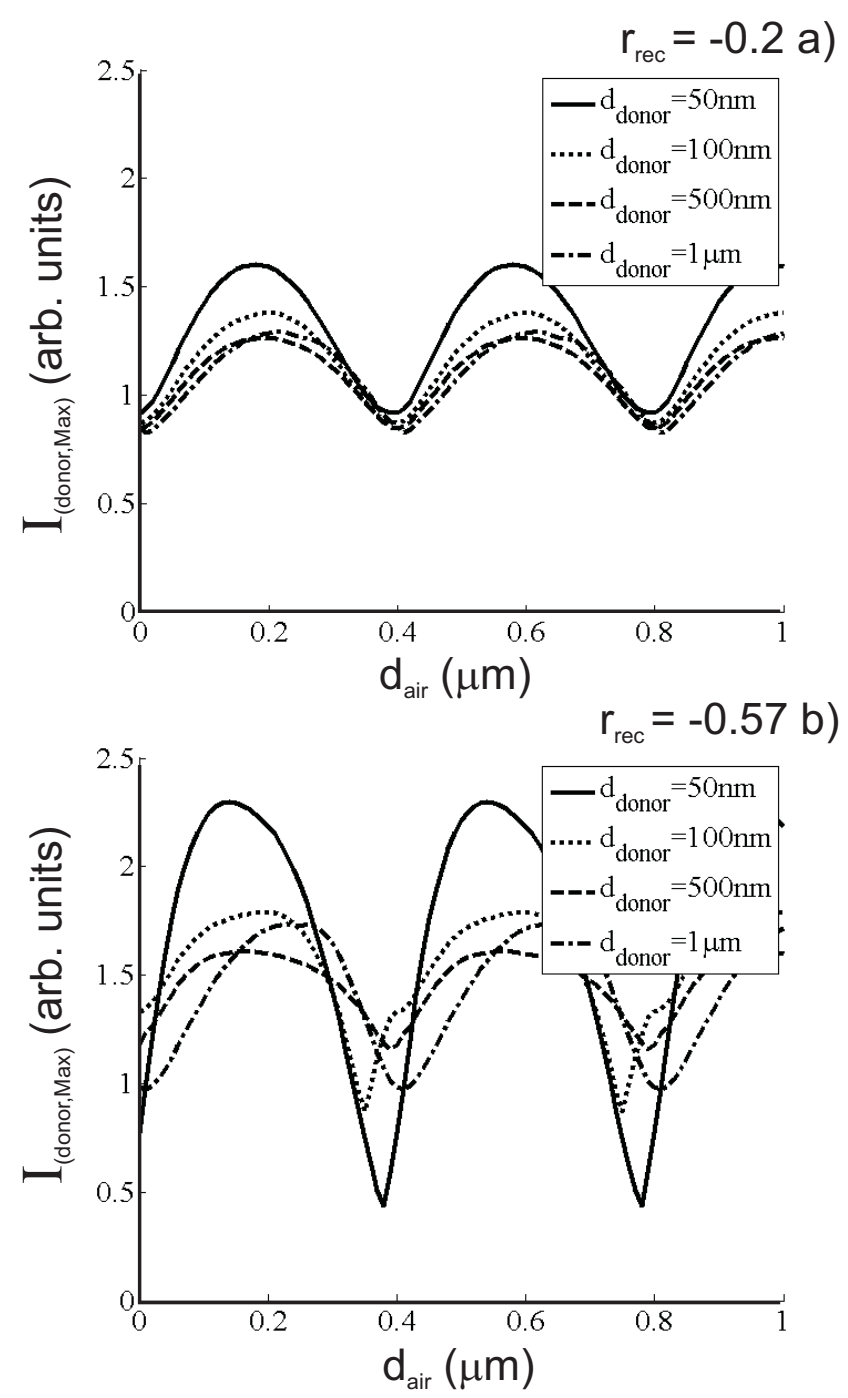

Fig. 4. Variation of the maximum intensity in the donor with $d_{a i r}$ and $d_{d o n o r}$ assuming $r_{r e c}=-0.2$ (a) and -0.57 (b). 


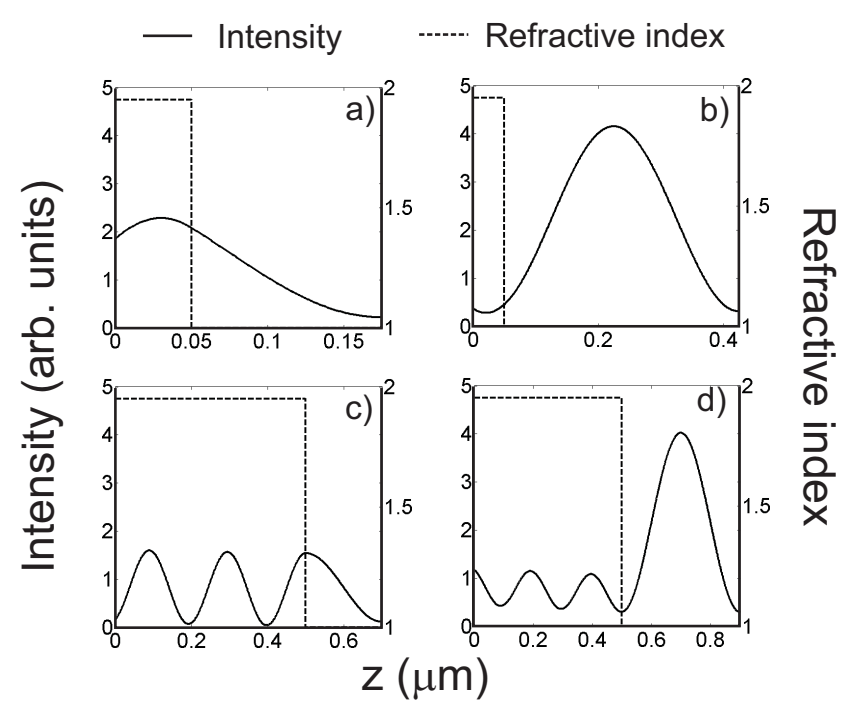

Fig. 5. Intensity (solid lines) and refractive index (dashed lines) profiles with $\left(d_{\text {donor }}(\mu m), d_{\text {air }}(\mu m)\right)$ equal to $(0.1,0.125),(0.1,0.375),(0.5,0.2)$, and $(0.5,0.4)$, in (a-d), respectively 

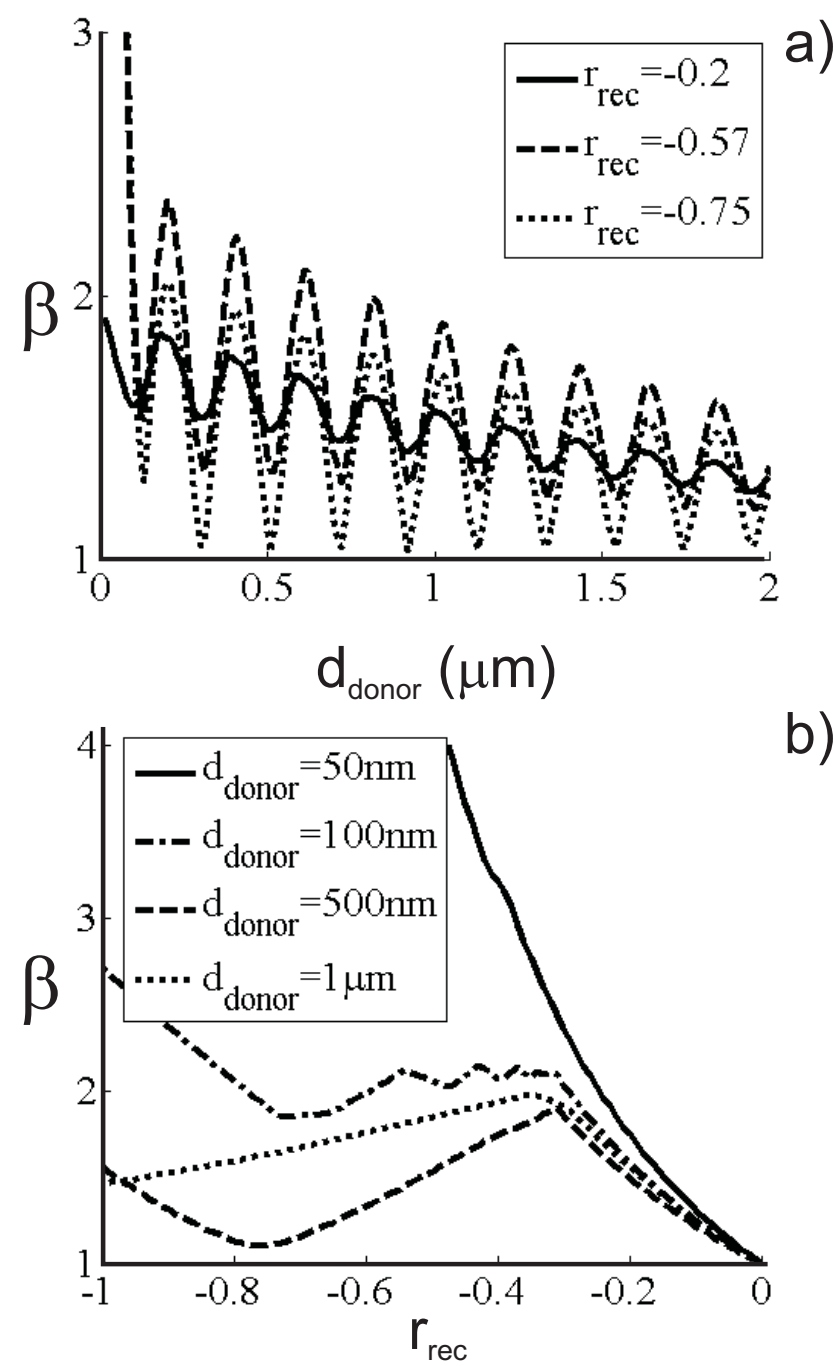

Fig. 6. Plot of $\beta$ as functions of $d_{\text {donor }}$ (a) and $r_{\text {rec }}$ (b) with other parameters fixed. 

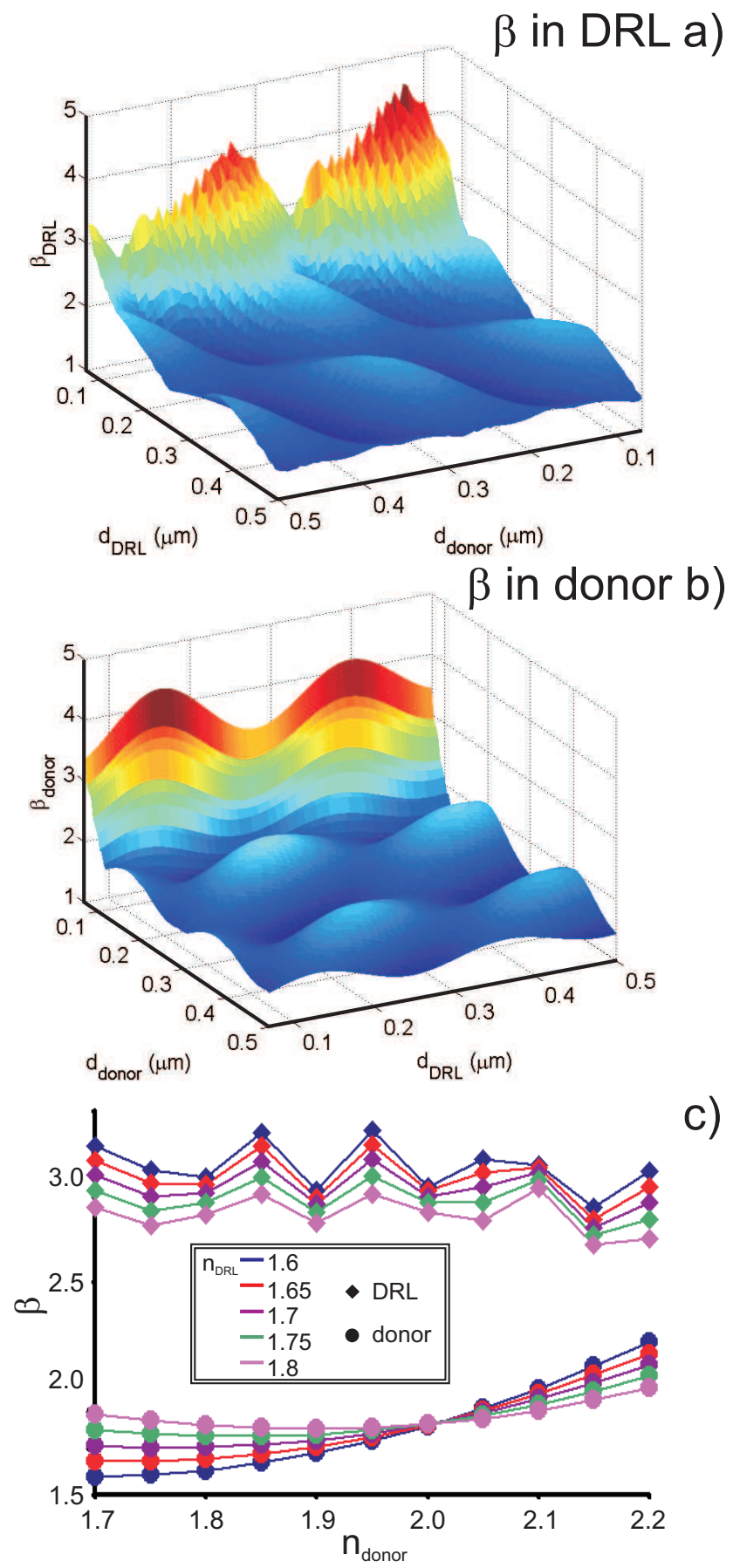

Fig. 7. Plots of $\beta$ in the TP-DRL (a) and in the GdGaO donor (b) as functions of $d_{\text {donor }}$ and $d_{D R L}$ with other parameters fixed, and variation of $\beta$ in the DRL (diamonds) and donor (circles) layers as function of $n_{D R L}$ and $n_{\text {donor }}$ (c). 


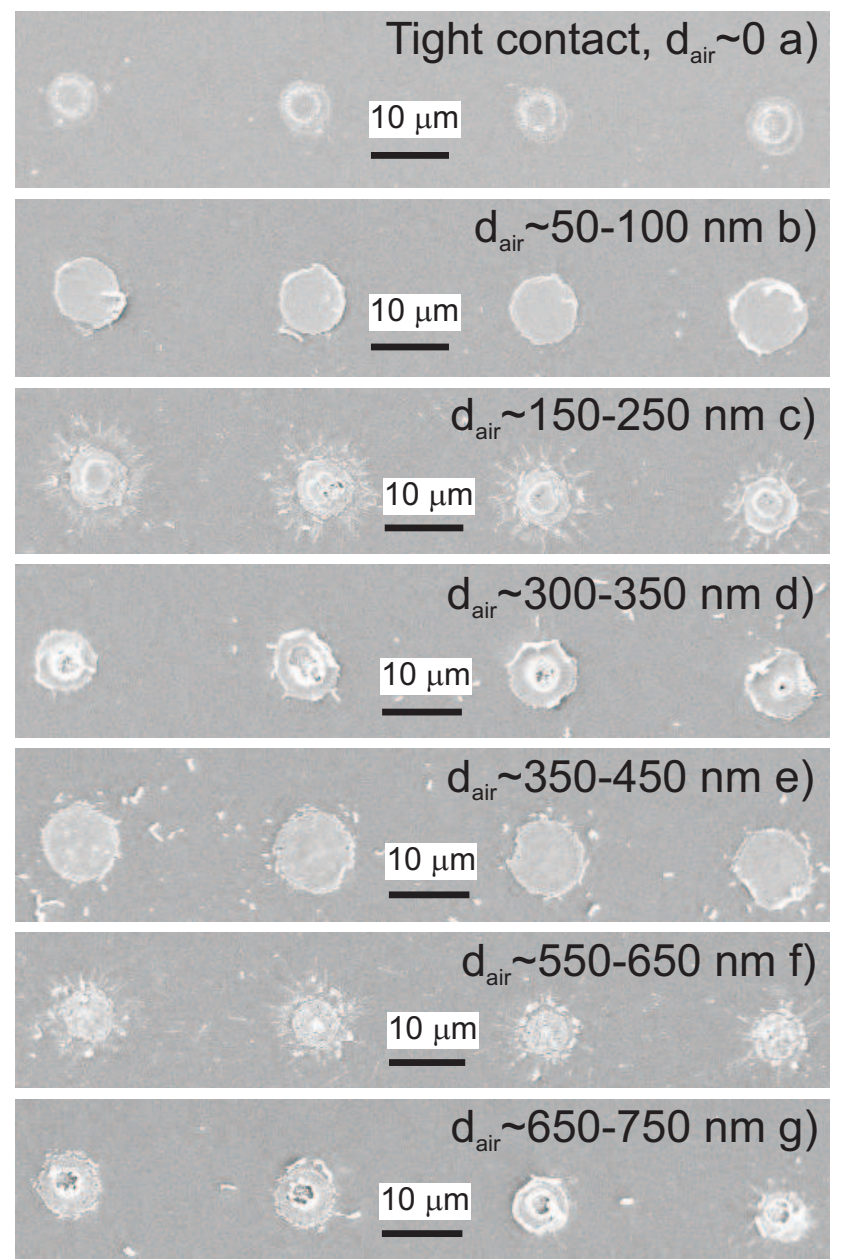

Fig. 8. SEM micrographs of $\mathrm{GdGaO}$ deposits on $\mathrm{Si}$ as a function of $d_{\text {air }}$ transferred with incident laser fluence $\approx 90 \mathrm{~mJ} / \mathrm{cm}^{2}$. 

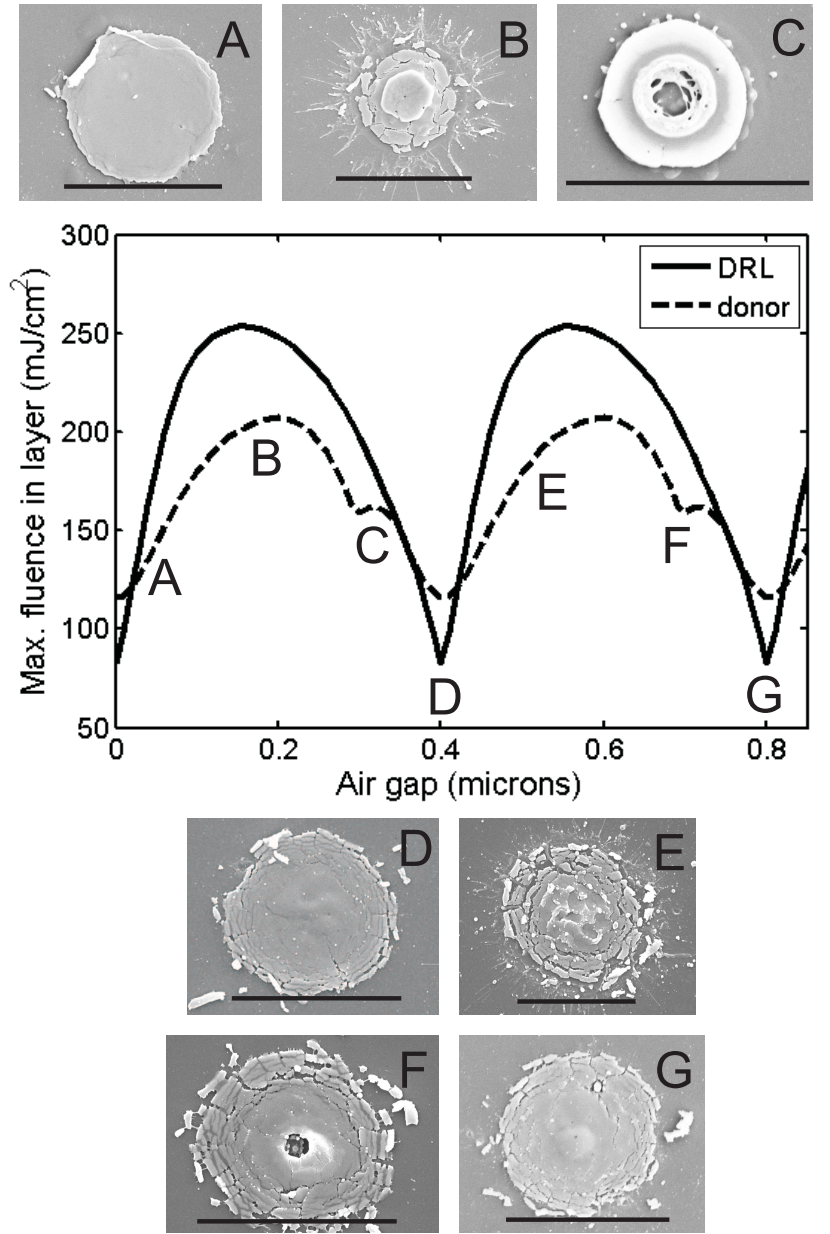

Fig. 9. Main graph: variation of maximum fluence in TP-DRL (solid line) and $\mathrm{GdGaO}$ donor (dashed line) as functions of $d_{\text {air }}$ assuming incident fluence 90 $\mathrm{mJ} / \mathrm{cm}^{2}$. SEM micrographs show deposited material at approximately indicated separations; scale bars represent $10 \mu \mathrm{m}$. 